PROCEEDINGS OF THE

AMERICAN MATHEMATICAL SOCIETY

Volume 127, Number 6, Pages 1711-1714

S 0002-9939(99)04708-5

Article electronically published on February 11, 1999

\title{
EXISTENCE OF MANY POSITIVE SOLUTIONS OF SEMILINEAR ELLIPTIC EQUATIONS ON AN ANNULUS
}

\author{
ZHI-QIANG WANG AND MICHEL WILLEM
}

(Communicated by Jeffrey B. Rauch)

\begin{abstract}
This paper is concerned with multiplicity of positive nonradial solutions of a nonlinear eigenvalue problem on an expanding annulus domain with a fixed width in $\mathbf{R}^{N}$ with $N \geq 4$. For $0<\lambda<\pi^{2}$, we show that the number of nonrotationally equivalent nonradial solutions tends to infinity as the inner radius of the domain tends to infinity.
\end{abstract}

In this paper, we study the existence of positive solutions of semilinear elliptic equations on an annulus in $\mathbf{R}^{N}, N \geq 4$ :

$$
\Omega_{r}:=\left\{x \in \mathbf{R}^{N}: r<|x|<r+1\right\},
$$

where $r>0$.

We consider the problem

$$
\begin{cases}-\Delta u=\lambda u+u^{2^{*}-1}, u>0 & \text { in } \Omega_{r}, \\ u=0 & \text { on } \partial \Omega_{r}\end{cases}
$$

where $2^{*}:=\frac{2 N}{N-2}$ is the critical Sobolev exponent.

Our main result is the following.

Theorem 1. For every $0<\lambda<\pi^{2}$ and $n \geq 1$, there exists $R(\lambda, n)$ such that for $r>R(\lambda, n)$ equation (1) has at least $n$ nonrotationally equivalent nonradial solutions.

For the corresponding subcritical problem (i.e., $2^{*}$ is replaced by some $p$ with $\left.2<p<2^{*}\right)$ the following result, which generalizes an earlier result of Coffman for $N=2([\mathrm{C}])$, is due to Y. Y. Li ([Ly]) and Suzuki ([S]) (see also $[\mathrm{K}]$ and $[\mathrm{Ls}]$ ): when $\lambda=0$, the number of nonrotationally equivalent nonradial solutions of the equation tends to infinity as $r$ tends to infinity. For the critical problem (1) with $\lambda=0$, it is also proved in [Ly] (see also [Ls] for some extensions) that there exists $r_{0}>0$ such that, for $r>r_{0},(1)$ has at least $\left[\frac{N}{2}\right]-1$ nonrotationally equivalent nonradial solutions.

Following the method introduced by Coffman in $[\mathrm{C}]$, we shall minimize the Rayleigh quotient on some spaces of invariant functions in $H_{0}^{1}\left(\Omega_{r}\right)$.

Let $G$ be a closed subgroup of $\mathbf{O}(\mathbf{N})$. The action of $G$ on $H_{0}^{1}\left(\Omega_{r}\right)$ is defined by

$$
g u(x):=u\left(g^{-1} x\right) .
$$

Received by the editors May 15, 1997 and, in revised form, September 10, 1997.

1991 Mathematics Subject Classification. Primary 35J20.

(C)1999 American Mathematical Society 
The subspace of invariant functions is defined by

$$
H_{0, G}^{1}\left(\Omega_{r}\right):=\left\{u \in H_{0}^{1}\left(\Omega_{r}\right): g u=u, \forall u \in G\right\} .
$$

For any $k \geq 1$, let $H_{k}$ be a representation of $\mathbf{Z}_{k}$ in $\mathbf{O}(\mathbf{2})$ defined by

$$
g z:=e^{\frac{i 2 \pi}{k}} z, \text { for } z \in \mathbf{C} \simeq \mathbf{R}^{2} .
$$

Then $H_{k}=\left\{g^{i}: i=0,1, \ldots, k-1.\right\}$. Define

$$
\begin{aligned}
G_{k} & :=H_{k} \times \mathbf{O}(\mathrm{N}-2), \\
G_{\infty} & :=\mathbf{O}(2) \times \mathbf{O}(\mathrm{N}-2) .
\end{aligned}
$$

Hence, $G_{k}$ has the canonical product action on $\mathbf{R}^{N}$.

We define also, for $k=1,2, \ldots, \infty$,

$$
S_{\lambda}(r, k):=\inf _{u \in H_{0, G_{k}}^{1}\left(\Omega_{r}\right),|u|_{2^{*}}=1} \int_{\Omega_{r}}|\nabla u|^{2}-\lambda u^{2},
$$

where $|u|_{2^{*}}$ is the $L^{2^{*}}$-norm of $u$.

The approach of [Ly] and [Ls] in the critical case is to choose $G$ in such a way that the embedding $H_{0, G}^{1}\left(\Omega_{r}\right) \subset L^{2^{*}}\left(\Omega_{r}\right)$ is compact. However, it seems impossible to obtain many solutions in this way. In order to overcome the difficulty, we use an idea in $[\mathrm{BN}]$ by Brezis and Nirenberg and a concentration-compactness result proved by P.L. Lions in [Lp].

Lemma 1. For every $0<\lambda<\pi^{2}$,

$$
\lim _{r \rightarrow \infty} S_{\lambda}(r, \infty)=\infty
$$

Proof. For every $r>0$ the Poincaré inequality

$$
\int_{\Omega_{r}}|\nabla u|^{2} \geq \lambda_{0}(r) \int_{\Omega_{r}} u^{2}
$$

holds on $H_{0}^{1}\left(\Omega_{r}\right)$ and

$$
\lim _{r \rightarrow \infty} \lambda_{0}(r)=\pi^{2} .
$$

Hence there exists $\delta>0$ and $r_{0}>0$ such that, for $r>r_{0}$ and $u \in H_{0}^{1}\left(\Omega_{r}\right)$,

$$
\int_{\Omega_{r}}|\nabla u|^{2}-\lambda u^{2} \geq\left(1-\frac{\lambda}{\lambda_{0}(r)}\right) \int_{\Omega_{r}}|\nabla u|^{2} \geq \delta \int_{\Omega_{r}}|\nabla u|^{2} .
$$

By Lemma 1.2' of [Ly],

$$
\lim _{r \rightarrow \infty} \inf _{u \in H_{0, G_{\infty}}^{1}\left(\Omega_{r}\right),|u|_{2^{*}=1}} \int_{\Omega_{r}}|\nabla u|^{2}=\infty .
$$

Lemma 2. For every $0<\lambda<\pi^{2}$ and $1 \leq k<\infty$,

$$
S_{\lambda}(r, k)=O(1), \text { as } r \rightarrow \infty .
$$

Proof. This result follows directly from Lemma 1.3 in [Ly].

Lemma 3. For every $0<\lambda<\pi^{2}$ there exists $r(\lambda)>0$ such that for every $1 \leq k \leq$ $\infty$ and $r>r(\lambda), S_{\lambda}(r, k)$ is attained by some function $u \in H_{0, G_{k}}^{1}\left(\Omega_{r}\right)$. 
Proof. If $k=\infty$, the result is a consequence of the compact embedding $H_{0, G_{\infty}}^{1}\left(\Omega_{r}\right) \subset$ $L^{\infty}\left(\Omega_{r}\right)$. For $1 \leq k<\infty$, we combine some arguments from Brezis-Nirenberg [BN] and P.L. Lions $[\mathrm{Lp}]$.

As in Lemma 1, there exists $\delta>0$ and $r(\lambda)>0$ such that, for $r>r(\lambda)$ and $u \in H_{0}^{1}\left(\Omega_{r}\right)$

$$
\int_{\Omega_{r}}|\nabla u|^{2}-\lambda u^{2} \geq \delta \int_{\Omega_{r}}|\nabla u|^{2} .
$$

Let $1 \leq k<\infty$ and $r>r(\lambda)$ be fixed. There is an open $B \subset \Omega_{r}$ such that

$$
g B \cap h B=\emptyset, \quad \forall g, h \in G_{k}, g \neq h .
$$

By a famous computation due to Brezis and Nirenberg [BN] (see also [Wm], Lemma 1.46) there exists a nonnegative $v \in H_{0}^{1}(B)$ such that $|v|_{2^{*}}=1$ and

$$
\int_{B}|\nabla v|^{2}-\lambda v^{2}<S
$$

where $S$ denotes the best Sobolev constant. It is clear that

$$
u=\sum_{g \in G_{k}} g v \in H_{0, G_{k}}^{1}\left(\Omega_{r}\right) .
$$

Moreover,

$$
\frac{\int_{\Omega_{r}}|\nabla u|^{2}-\lambda u^{2}}{\left(\int_{\Omega_{r}} u^{2^{*}}\right)^{\frac{2}{2^{*}}}}<k^{\frac{2}{N}} S,
$$

so that

$$
S_{\lambda}(r, k)<k^{\frac{2}{N}} S .
$$

By a result of P. L. Lions ([Lp], see also [Wm], Theorem 8.15, and [Wz]), $S_{\lambda}(r, k)$ is attained by some $u \in H_{0, G_{k}}^{1}\left(\Omega_{r}\right)$.

Lemma 4. For every $0<\lambda<\pi^{2}, r>r(\lambda)$ (which is given in Lemma 3), $k \geq 1$ and $m \geq 2$, if $S_{\lambda}(r, \mathrm{~km})$ is achieved and

$$
S_{\lambda}(r, k m)<S_{\lambda}(r, \infty)
$$

then

$$
S_{\lambda}(r, k)<S_{\lambda}(r, k m) .
$$

Proof. This can be proved with essentially the same proof as of Lemma 1.5 in [Ly].

Proof of Theorem 1. Let $0<\lambda<\pi^{2}$ and $n \geq 1$ be fixed. According to Lemmas 1 and 2, there exists $R(\lambda, n)$ such that

$$
S_{\lambda}\left(r, 2^{n}\right)<S_{\lambda}(r, \infty)
$$

for $r>R(\lambda, n)$. We can assume that $R(\lambda, n)>r(\lambda)$, so that $S_{\lambda}(r, k)$ is achieved for $1 \leq k \leq \infty$ and $r>R(\lambda, n)$ by Lemma 3. Lemma 4 implies that

$$
S_{\lambda}(r, 2)<S_{\lambda}\left(r, 2^{2}\right)<\cdots<S_{\lambda}\left(r, 2^{n}\right)<S_{\lambda}(r, \infty)
$$

for $r>R(\lambda, n)$. 
Therefore, all minimizers for $S_{\lambda}\left(r, 2^{j}\right)$ are nonradial and nonrotationally equivalent. We may assume that the minimizers are nonnegative. By the symmetric criticality principle (see e.g. $[\mathrm{Wm}]$ ) the minimizers, after rescaling, are solutions of

$$
-\Delta u-\lambda u=u^{2^{*}-1}, \text { in } \Omega_{r} .
$$

The maximum principle implies that $u>0$ in $\Omega_{r}$.

We remark that we do not know whether our result holds for $N=3$. Note that it is also open for the subcritical problem of whether the multiplicity results in [Ly] (also $[\mathrm{C}],[\mathrm{K}],[\mathrm{Ls}],[\mathrm{S}]$ ) hold for $N=3$.

\section{ACKNOWLEDGMENT}

We thank the referee for some useful remarks. The first author is partially supported by an NSF grant.

\section{REFERENCES}

[BN] Brezis H. \& Nirenberg L., Positive solutions of nonlinear elliptic equations involving critical exponents, Comm. Pure Appl. Math. 36 (1983), 437-477. MR 84h:35059

[C] C.V. Coffman, A nonlinear boundary value problem with many positive solutions, J. Diff. Equa., 54 (1984), 429 - 637. MR 86e:35055

$[\mathrm{K}] \quad$ B. Kawohl, "Rearrangements and Convexity of Level Sets in P. D. E.", Lecture Notes in Math. 1150, Springer 1985. MR 87a:35001

[Ly] Y.Y. Li, Existence of many positive solutions of semilinear elliptic equations on annulus, J. Diff. Equa., 83 (1990), 348-367. MR 91a:35073

[Ls] S.S. Lin, Existence of many positive nonradial solutions for nonlinear elliptic equations on an annulus, J. Diff. Equa., 103 (1993), 338-349. MR 94k:35108

[Lp] P.L. Lions, Symmetry and the concentration-compactness method, In Marino at al, editors, Nonlinear Variational Problems, 47-56. Pitman, 1985. MR 86m:49011

[S] T. Suzuki, Radial and nonradial solutions for semilinear elliptic equations on circular domains, Ed. G. Talenti, Simposia mathematica, 30(1989), 153-174. MR 91k:35022

$[\mathrm{Wz}]$ Z.-Q. Wang, Construction of multi-peaked solutions for a nonlinear Neumann problem with critical exponent in symmetric domains, Nonlinear Anal. TMA, 27(1996), 1281 - 1306. MR 97g:35056

[Wm] M. Willem, "Minimax Theorems", Birkhäuser, 1996. MR 97h:58037

Department of Mathematics, Utah State University, Logan, Utah 84322-3900

E-mail address: wang@math.usu.edu

Institut de Mathématique Pure et Appliquée, Université Catholique de Louvain, B1348 Louvain-La-Neuve, Belgium 